\title{
Heat transfer in free convection flow of micropolar fluids over an oscillating vertical plate
}

\author{
Asma Khalid a, Ilyas Khan b, Sharidan Shafie c," \\ a Department of Mathematics, SBK Women's University, Quetta 87300, Pakistan \\ ${ }^{b}$ College of Engineering Majmaah University, Majmaah 11952, Saudi Arabia \\ c Department of Mathematical Sciences, Faculty of Science, Universiti Teknologi Malaysia, 81310 UTM Johor Bahru, Mayalsia \\ Johor, Malaysia \\ *Corresponding author: sharidan@utm.my
}

\section{Article history}

Received 29 August 2017

Accepted 30 November 2017

\section{Graphical abstract}

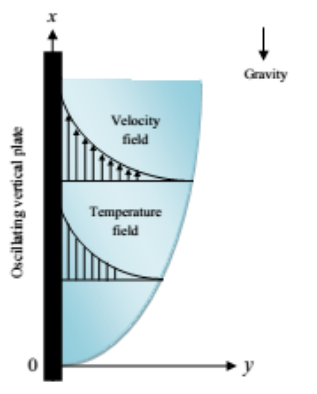

\begin{abstract}
An analytical investigation is carried out to study the unsteady free convection stream of micropolar fluids over an oscillating vertical plate. Wall couple stress is promised at the bounding plate with isothermal temperature. Problem is displayed in terms of combined partial differential equations together with certain physical conditions and then written in non-dimensional form. Exact solutions are achieved using the Laplace transform technique. Analytical results of velocity, microrotation and temperature are plotted in graphs and debated for different inserted parameters. Excellent validation of present results is acquired with existing results in literature. It is observed that, the velocity is smaller for micropolar fluids than for Newtonian fluids.
\end{abstract}

Keywords: Micropolar fluids, microrotaions, free convection, exact solutions

\section{INTRODUCTION}

Rheological characteristics of non-Newtonian fluids are termed by their constitutive equations. Because of complex nature of nonNewtonian fluids, several models or constitutive equations have been suggested based on their empirical observations. Amongst them, one model was proposed by (Eringen, 1966) using micromorphic fluid theory, known as micropolar fluids. Micropolar fluids pose microrotation and microinertia effect and constitute a significant branch of fluids. In literature micropolar fluids are used to describe flow characteristics of colloidal suspensions, geomor-phological sediments, liquid crystals, polymeric additives, haematological suspensions, lubricants and many other biological fluids (Ariman et al., 1973). Eringen and Lukaszewicz discussed fascinating characteristics of theory and applications of micropolor fluids (Erigen, 2001; Lukaszewicz 1999).

(Nazar et al., 2002) examined boundary layer flow of micropolar fluid past an isothermal sphere by taking the free convection. (Cheng, 2008) discussed natural convection for micropolar fluids in combined transfer of heat and mass over a sphere accepting constant wall temperature together with constant wall concentration. (Sherief et al., 2011) used Laplace transform method and studied unsteady flow of a micropolar fluid from with rapidly stimulated plate. Analysis of heat transfer from moving surfaces with internal heat generation in a micropolar fluid is carried out by (El-Hakiem, 2014 ). (Hassanien et al., 1997) investigated the natural convection boundary layer flow of a micropolar fluid. (Ishak et al., 2008) discussed the micropolar fluids with heat transfer over a stretching surface with variable heat flux. (Lok et et al., 2005) reported the steady mixed convection flow near the stagnation point on a vertical surface of a micropolar fluid. Boundary layer stagnation point flow past a moving wall of a micropolar fluid has been studied by (Gorla, 1983). (Damesh et al., 2007) illustrated the micropolar fluid with unsteady natural convection heat transfer over a vertical surface with constant heat flux using numerical technique, whereas conjugate transfer of heat and mass in unsteady flow of a micropolar fluids with wall couple stress studied by (Khalid et al., 2015).

(Abo-Dahab et al., 2013) investigated the unsteady flow of rotating and chemically reacting slip-flow regime with heat generation MHD micropolar fluid. The aim of the present work is to provide exact solutions for the unsteady free convection flow of an incompressible micropolar fluid over an infinite vertical plate oscillating in its own plan. More exactly, heat transfer when the bounding plate takes wall couple stress with isothermal temperature is studied.

\section{PROBLEM FORMULATION}

Assume, unsteady boundary layer stream of an incompressible micropolar fluid in the region $y>0$ determine by a plane surface positioned at $y=0$ with a fixed end at $x=0$. It is expected that at the initial moment $t=0$, both the plate and the fluid are at rest at the constant temperature $T_{\infty}$. At time $t=0^{+}$the plate begins to oscillate in its plane $(y=0)$ according to 


$$
\boldsymbol{V}=U H(t) \cos (\omega t) \mathbf{i} ; t>0
$$

where $H(t)$ is the unit step function, $U$ is the amplitude of the motion, $\mathbf{i}$ is the unit vector in the vertical flow direction and $\omega$ is the frequency of oscillation of the plate. At the same time, the plate temperature level are raised to $T_{w}$ which are thereafter maintained constants. Assume that, the velocity, microrotation and temperature are functions of $y$ and $t$ only. Taking usual Boussinesq's approximation, the unsteady flow is governed by the following set of partial differential equations.

$$
\begin{gathered}
\rho \frac{\partial u}{\partial t}=(\mu+\alpha) \frac{\partial^{2} u}{\partial y^{2}}+\alpha \frac{\partial N}{\partial y}+\rho g \beta_{f}\left(T-T_{\infty}\right), \\
\rho j \frac{\partial N}{\partial t}=\gamma_{0} \frac{\partial^{2} N}{\partial y^{2}}, \\
\rho c_{p} \frac{\partial T}{\partial t}=k \frac{\partial^{2} T}{\partial y^{2}} .
\end{gathered}
$$

The corresponding initial and boundary conditions are

$$
\begin{aligned}
& u(y, 0)=0, N(y, 0)=0, T(y, 0)=T_{\infty} \text { for all } y \geq 0, \\
& u(0, t)=H(t) U \cos (\omega t), N(0, t)=-n \frac{\partial u}{\partial y}(0, t),(6) \\
& T_{\infty}(0, t)=T_{w}, t>0, \\
& u(\infty, t) \rightarrow 0, N(\infty, t) \rightarrow 0, T(\infty, t) \rightarrow T_{\infty} \text { as } y \rightarrow \infty .
\end{aligned}
$$

Here $u$ is velocity, $\mu$ is dynamic viscosity, $\rho$ is density, $g$ is gravitational acceleration, $\alpha$ is vortex viscosity, $t$ is time, $T$ is temperature, $\beta_{T}$ is volumetric coefficient of thermal expansion, $N$ is the microrotation whose direction of rotation is in the $x y$-plane, $j$ is microinertia per unit mass, $\gamma_{0}$ is spin gradient viscosity, $c_{p}$ is heat capacity at constant pressure, $k$ is thermal conductivity and $\omega t$ is phase angle. The spin gradient viscosity $\gamma_{0}$, measures the relationship between the coefficients of viscosity and micro-inertia, is defined as

$$
\gamma_{0}=\left(\mu+\frac{\alpha}{2}\right) j
$$

To moderate the above equations (2-8) into their non-dimensional forms, we establish the succeeding non-dimensional quantities,

$$
\begin{aligned}
& y^{*}=\frac{U}{v} y, t^{*}=\frac{U^{2}}{v} t, u^{*}=\frac{u}{U}, N^{*}=\frac{v}{U^{2}} N, \\
& \theta=\frac{T-T_{\infty}}{T_{w}-T_{\infty}}, \omega^{*}=\frac{v}{U^{2}} \omega, j^{*}=\frac{U^{2}}{v^{2}} j .
\end{aligned}
$$

Employing equation (8) into equations (2-4), we achieve the following non-dimensional partial differential equations $\left({ }^{*}\right.$ symbol is omitted for simplicity)

$$
\begin{gathered}
\frac{\partial u}{\partial t}=(1+\beta) \frac{\partial^{2} u}{\partial y^{2}}+\beta \frac{\partial N}{\partial y}+G r \theta \\
\frac{\partial N}{\partial t}=\frac{1}{\eta} \frac{\partial^{2} N}{\partial y^{2}} \\
\operatorname{Pr} \frac{\partial \theta}{\partial t}=\frac{\partial^{2} \theta}{\partial y^{2}}
\end{gathered}
$$

The consistent initial and boundary conditions take the following nondimensional forms:

$$
\begin{aligned}
& u(y, 0)=0, N(y, 0)=0, \theta(y, 0)=0 \text { for all } y \geq 0, \\
& u(0, t)=H(t) \cos (\omega t), N(0, t)=-n \frac{\partial u}{\partial y}(0, t) \\
& \theta(0, t)=1, t>0, \\
& u(\infty, t) \rightarrow 0, N(\infty, t) \rightarrow 0, \theta(\infty, t) \rightarrow 0 \text { as } y \rightarrow \infty
\end{aligned}
$$

where

$$
\beta=\frac{\alpha}{\mu}, \operatorname{Pr}=\frac{\mu c_{p}}{k}, \eta=\frac{\mu j}{\gamma_{0}}, G r=\frac{g \beta_{T}\left(T_{w}-T_{\infty}\right) v}{U^{3}},
$$

are the microrotation parameter, Prandtl number, dimensionless spin gradient and Grashof number, respectively.

\section{SOLUTION OF THE PROBLEM}

Applying the Laplace transforms to equations (9-11), and yusing conditions (12-14), the following solutions in the transformed

plane are obtained

$$
\begin{aligned}
\bar{u}(y, q)= & a_{4} \frac{q}{\left(q^{2}+\omega^{2}\right)} e^{-y \sqrt{\beta_{0} q}}+a_{5} \frac{q}{\left(q^{2}+\omega^{2}\right)} e^{-y \sqrt{\eta q}} \\
& +a_{6} \frac{1}{q^{2}} e^{-y \sqrt{\beta_{0} q}}+a_{7} \frac{1}{q^{2}} e^{-y \sqrt{\eta q}} \\
& -a_{8} \frac{1}{q^{2}} e^{-y \sqrt{\operatorname{Pr} q}}
\end{aligned}
$$

$$
\begin{gathered}
\bar{N}(y, q)=\frac{a_{1}}{\sqrt{q}} e^{-y \sqrt{\eta q}}-\frac{a_{1} \omega}{2 i} \frac{1}{\sqrt{q}(q+i \omega)} e^{-y \sqrt{\eta q}} \\
+\frac{a_{1} \omega}{2 i} \frac{1}{\sqrt{q}(q-i \omega)} e^{-y \sqrt{\eta q}}+\frac{a_{2}}{q \sqrt{q}} e^{-y \sqrt{\eta q}}, \\
\bar{\theta}(y, q)=\frac{1}{q} e^{-y \sqrt{\operatorname{Pr} q}} .
\end{gathered}
$$

By taking the inverse Laplace transforms of above equations, we obtain

$$
\begin{gathered}
u(y, t)=u_{1}(y, t)+u_{2}(y, t)-u_{3}(y, t)-u_{4}(y, t) \\
+u_{5}(y, t)+u_{6}(y, t)-u_{7}(y, t), \\
N(y, t)=N_{1}(y, t)+N_{2}(y, t), \\
\theta(y, t)=\theta_{1}(y, t),
\end{gathered}
$$

with

$$
\begin{aligned}
& u_{1}(y, t)=\left(\frac{a_{4}}{4}\right) H(t) e^{-i \omega t}\left[\begin{array}{l}
e^{-y \sqrt{-i \omega \beta_{0}}} \operatorname{erfc}\left(\frac{y}{2} \sqrt{\frac{\beta_{0}}{t}}-\sqrt{-i \omega t}\right) \\
+e^{y \sqrt{-i \omega \beta_{0}}} \operatorname{erfc}\left(\frac{y}{2} \sqrt{\frac{\beta_{0}}{t}}+\sqrt{-i \omega t}\right)
\end{array}\right], \\
& u_{2}(y, t)=\left(\frac{a_{4}}{4}\right) H(t) e^{i \omega t}\left[\begin{array}{l}
e^{-y \sqrt{i \omega \beta_{0}}} \operatorname{erfc}\left(\frac{y}{2} \sqrt{\frac{\beta_{0}}{t}}-\sqrt{i \omega t}\right) \\
+e^{y \sqrt{i \omega \beta_{0}}} \operatorname{erfc}\left(\frac{y}{2} \sqrt{\frac{\beta_{0}}{t}}+\sqrt{i \omega t}\right)
\end{array}\right],
\end{aligned}
$$




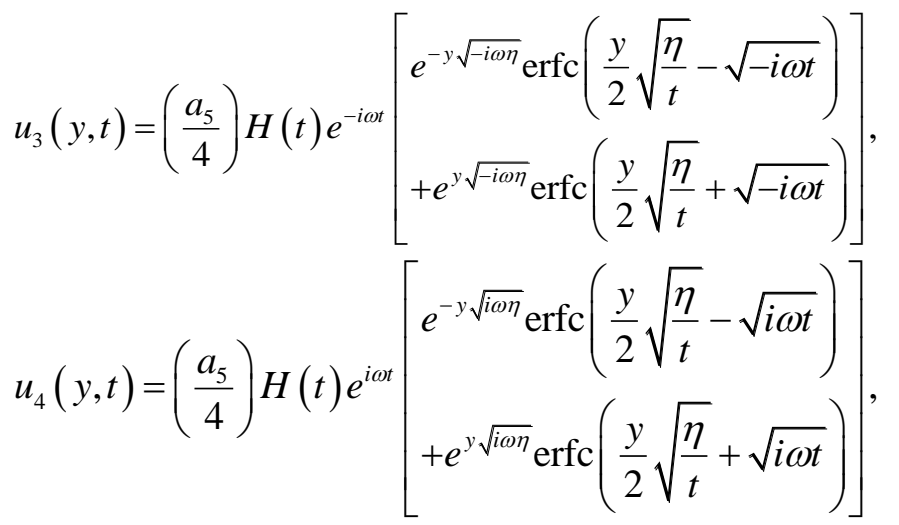

$u_{5}(y, t)=a_{6}\left[\left(t+\frac{y^{2} \beta_{0}}{2}\right) \operatorname{erfc}\left(\frac{y}{2} \sqrt{\frac{\beta_{0}}{t}}\right)-y \sqrt{\beta_{0}} \sqrt{\frac{t}{\pi}} e^{-\frac{y^{2} \beta_{0}}{4 t}}\right]$,

$u_{6}(y, t)=a_{7}\left[\left(t+\frac{y^{2} \eta}{2}\right) \operatorname{erfc}\left(\frac{y}{2} \sqrt{\frac{\eta}{t}}\right)-y \sqrt{\eta} \sqrt{\frac{t}{\pi}} e^{-\frac{y^{2} \eta}{4 t}}\right]$,

$u_{7}(y, t)=a_{8}\left[\left(t+\frac{y^{2} \operatorname{Pr}}{2}\right) \operatorname{erfc}\left(\frac{y}{2} \sqrt{\frac{\operatorname{Pr}}{t}}\right)-y \sqrt{\operatorname{Pr}} \sqrt{\frac{t}{\pi}} e^{-\frac{y^{2} \operatorname{Pr}}{4 t}}\right]$,

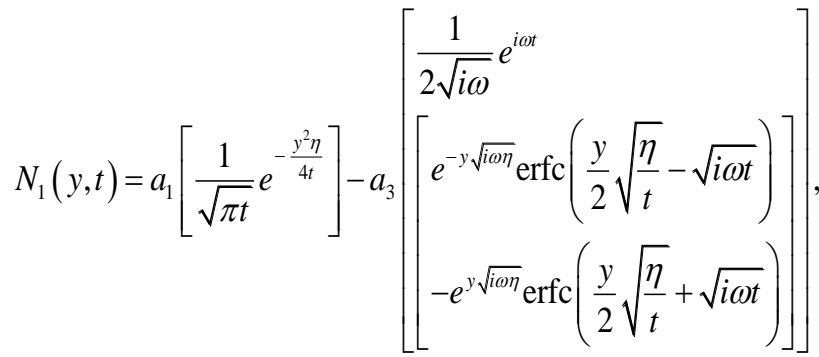

$N_{2}(y, t)=a_{3}\left[\begin{array}{l}\frac{1}{2 \sqrt{-i \omega}} e^{-i \omega t} \\ e^{-y \sqrt{-i \omega \eta}} \operatorname{erfc}\left(\frac{y}{2} \sqrt{\frac{\eta}{t}}-\sqrt{-i \omega t}\right) \\ -e^{y \sqrt{-i \omega \eta}} \operatorname{erfc}\left(\frac{y}{2} \sqrt{\frac{\eta}{t}}+\sqrt{-i \omega t}\right)\end{array}\right]$

$+a_{2}\left[2 \sqrt{\frac{t}{\pi}} e^{-\frac{y^{2} \eta}{4 t}}-y \sqrt{\eta} \operatorname{erfc}\left(\frac{y}{2} \sqrt{\frac{\eta}{t}}\right)\right]$,

$\theta_{1}(y, t)=\operatorname{erfc}\left(\frac{y}{2} \sqrt{\frac{\operatorname{Pr}}{t}}\right)$,

where

$$
\begin{aligned}
& a_{1}=\frac{\left(n \sqrt{\beta_{0}}\right)\left(\eta-\beta_{0}\right)}{\left(\eta-\beta_{0}+n \beta \beta_{0} \sqrt{\eta \beta_{0}}-n \beta_{0} \beta \eta\right)}, \\
& a_{2}=A_{1} n G r \sqrt{\beta_{0}} \frac{\left(\eta-\beta_{0}\right)}{\left(\eta-\beta_{0}+n \beta \beta_{0} \sqrt{\eta \beta_{0}}-n \beta_{0} \beta \eta\right)} \\
& -A_{1} n G r \sqrt{\operatorname{Pr}} \frac{\left(\eta-\beta_{0}\right)}{\left(\eta-\beta_{0}+n \beta \beta_{0} \sqrt{\eta \beta_{0}}-n \beta_{0} \beta \eta\right)},
\end{aligned}
$$

$$
\begin{aligned}
& a_{3}=\frac{a_{1} \omega}{2 i}, \quad a_{4}=1-A_{0} a_{1}, \\
& a_{5}=A_{0} a_{1}, \quad a_{6}=G r A_{1}-a_{2} A_{0}, \\
& a_{7}=a_{2} A_{0}, \quad a_{8}=G r A_{1}, \\
& A_{0}=\frac{\beta \beta_{0} \sqrt{\eta}}{\eta-\beta_{0}}, \quad A_{1}=\frac{\beta_{0}}{\operatorname{Pr}-\beta_{0}}, \\
& \beta_{0}=\frac{1}{1+\beta} .
\end{aligned}
$$

\section{Limiting case}

In this case, the stream in the fluid is convinced due to spontaneous motion of the plate. Hence taking $\omega t=\beta=\eta=0$ into equation (18), which correspond the spontaneous motion of the plate as

$$
\begin{aligned}
u(y, t) & =\frac{1}{4}\left[\operatorname{erfc}\left(\frac{y}{2 \sqrt{t}}\right)\right] \\
& +a_{6}\left[\left(t+\frac{y^{2}}{2}\right) \operatorname{erfc}\left(\frac{y}{2 \sqrt{t}}\right)-y \sqrt{\frac{t}{\pi}} e^{-\frac{y^{2}}{4 t}}\right] \\
& -a_{8}\left[\begin{array}{l}
\left.\left(t+\frac{y^{2} \operatorname{Pr}}{2}\right) \operatorname{erfc}\left(\frac{y}{2} \sqrt{\frac{\operatorname{Pr}}{t}}\right)\right] . \\
-y \sqrt{\operatorname{Pr}} \sqrt{\frac{t}{\pi}} e^{-\frac{y^{2} \operatorname{Pr}}{4 t}}
\end{array}\right]
\end{aligned}
$$

It is important to note that equation (21) is found identical to those obtained by (Chudhary et al., 2007) equation (19), in the absence of MHD and porosity effects. Hence, this verifies the correctness of the present work. This fact is also shown in Figure 7.

\section{RESULTS AND DISCUSSION}

In this section, final results are computed for different physical parameters which are presented by mean of graphs. Parameters of physical interest are microrotation parameter $\beta$, Prandtl number $\operatorname{Pr}$ and Grashof number $G r$. Influence of microrotation parameter $\beta$ on velocity and the microrotation is depicted in Fig.s 1 and 2 . These graphs show that velocity decreases whereas microrotation increases with increasing $\beta$. Obviously, velocity satisfies the imposed boundary conditions in equations (13) and (14). On the other hand, the microrotation takes the negative values of the gradient of velocity at the plate surface and approaching to zero as one move away from the plate surface as shown in Fig. 2. This fact totally aggresses with imposed conditions on microrotation (see equations (13) and (14)). Fig.s 3 and 4 present plots for velocity and microrotation for different values of Prandtl number Pr. These graphs show that the influence of increasing values of Pr result in decreasing of the velocity, magnitude of the microrotation and temperature as well.

Fig.s 5 and 6 show deviations in velocity and microrotation outlines for different values of Grashof number Gr. It is observed that an increase in $G r$ leads to an increase in velocity due to enhancement in the buoyancy force. Besides that magnitude of microrotation decreases for large values of $G r$. For positive values of $G r$ correspond to freezing of the surface by natural convection however $G r=0$ shows the lack of heat transfer due to free convection. 


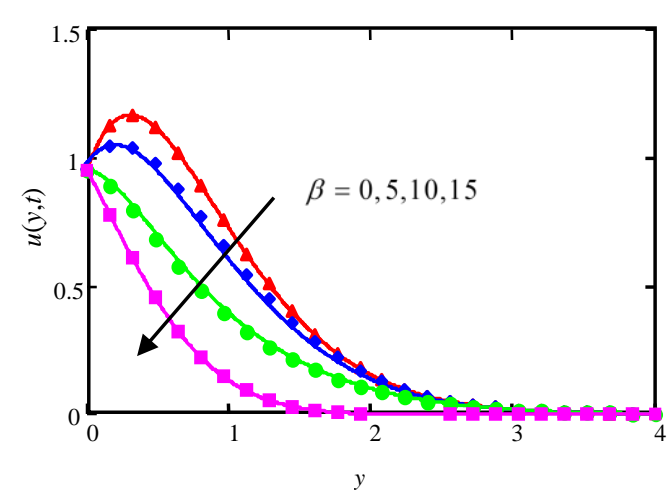

Fig. 1 Velocity profiles for different values of $\beta$ when $\operatorname{Pr}=10, \eta=1.5, n=0.6, G r=5, \omega=\pi / 4 \& t=0.6$.

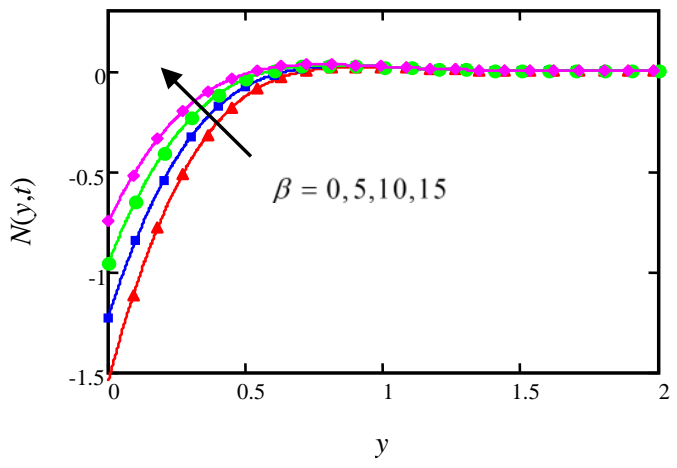

Fig. 2 Microrotations for different $\beta$ when $\operatorname{Pr}=10, \eta=1.5, n=0.6, G r=5, \omega=\pi / 4 \& t=0.6$.

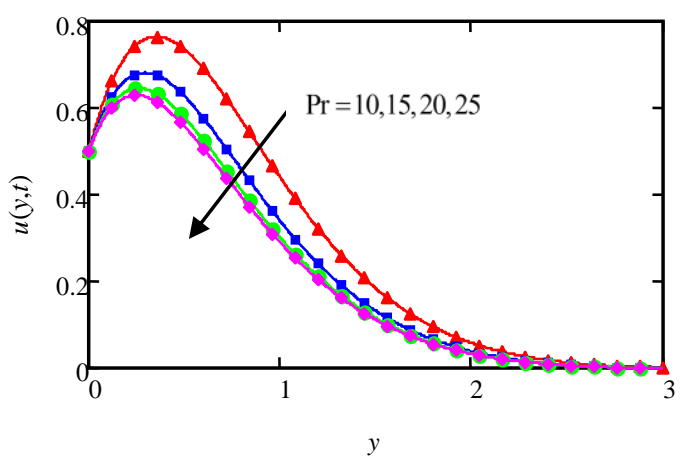

Fig. 3 Velocity profiles for different values of $\operatorname{Pr}$ when $\beta=0.5, \eta=1.5, n=0.6, G r=5, \omega=\pi / 4 \& t=0.6$.

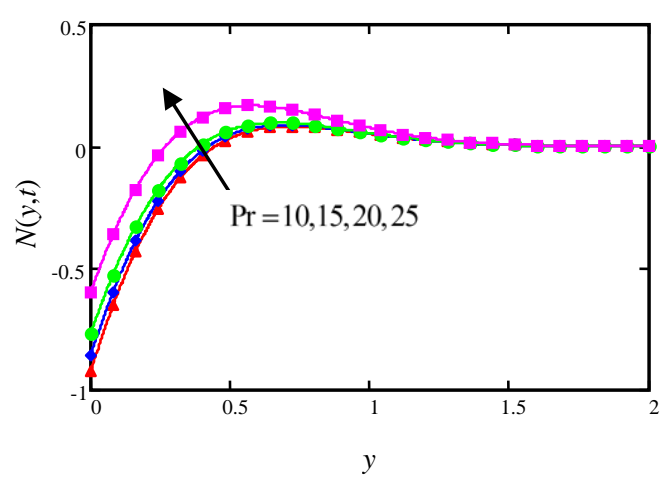

Fig. 4 Microrotations for different $\operatorname{Pr}$ when $\beta=0.5, \eta=1.5, n=0.6, G r=5, \omega=\pi / 4 \& t=0.6$.

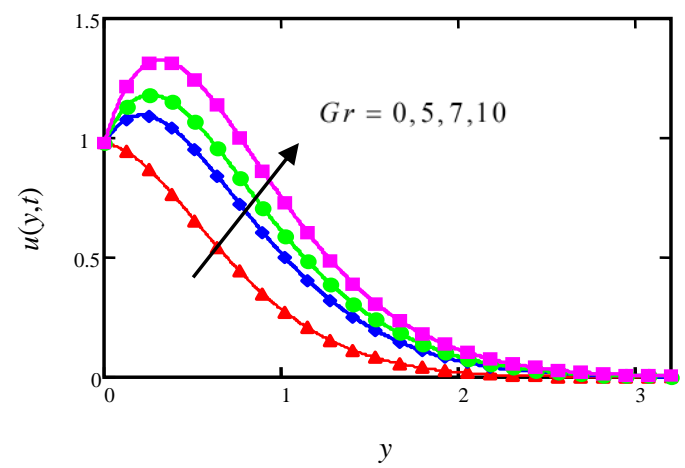

Fig. 5 Velocity profiles for different $\mathrm{Gr}$ when $\operatorname{Pr}=10, \beta=0.5, \eta=1.5, n=0.6, \omega=\pi / 4 \& t=0.6$.

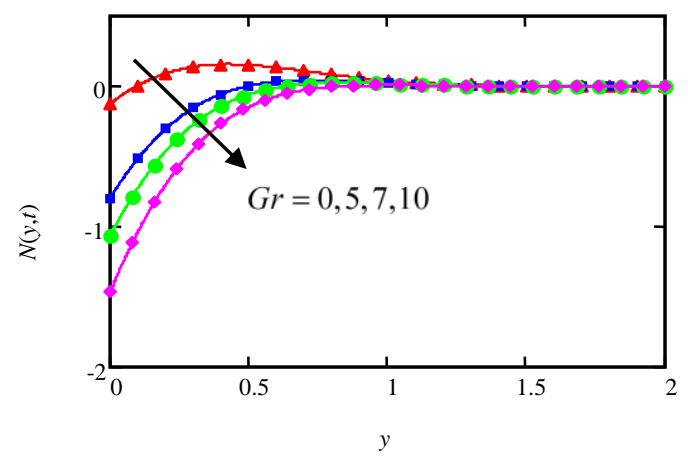

Fig. 6 Microrotaions for different $\mathrm{Gr}$ when $\operatorname{Pr}=10, \beta=0.5, \eta=1.5, n=0.6, \omega=\pi / 4 \& t=0.6$. 
In order to check the correctness of present results, the velocity profiles of present result, equation (21) is compared with existing results in literature (Chaudhary et al., 2007). This comparison is shown in Fig. 7.

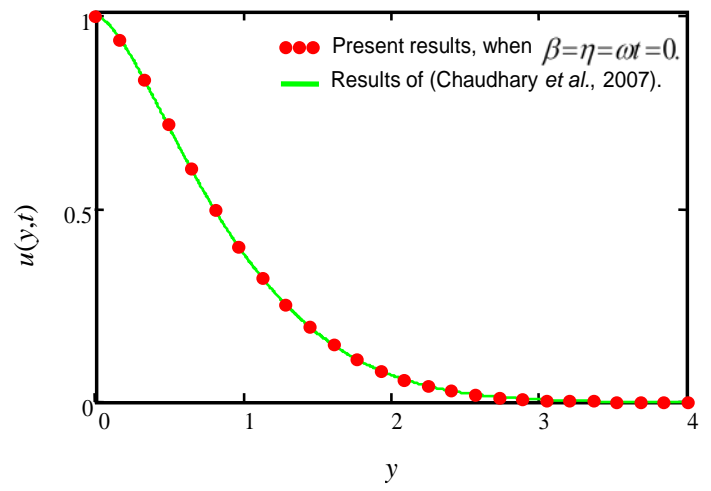

Fig. 7 Comparison of micropolar fluid velocity (when $\beta=\eta=0$ ), with results obtained by (Chaudhary et al., 2007) see Equation (19), when $t=0.2, \omega t=0$ and $M^{\prime}=0$.

\section{CONCLUSION}

An analytical study is performed to study explore the unsteady free convection stream of micropolar fluids over an oscillating vertical plate with wall couple stress. Problem is demonstrated in terms of coupled partial differential equations together with some physical conditions and then written in non-dimensional form. Laplace transform technique is used to find the exact solutions. It is concluded that velocity across the boundary layer increases with increasing $\mathrm{Pr}$ whereas decreases with increasing values of $\beta$ and Pr. Magnitude of microrotation on the plate is decreases with increasing $G r$ while rises with increasing $\beta$ and Pr. Solution (21) is found in excellent agreement with those obtain by (Chaudhary et al., 2007).

\section{ACKNOWLEDGEMENT}

The authors would like to acknowledge the Ministry of Education Malaysia (MOE) and Research Management Centre-UTM for the financial support through vote numbers $06 \mathrm{H} 67,4 \mathrm{~F} 538,4 \mathrm{~F} 713$ and $15 \mathrm{H} 80$ for this research.

\section{REFERENCES}

Nur, H., Hayati, F., Hamdan, H. 2007. On the location of different titanium sites in Ti-OMS-2 and their catalytic role in oxidation of styrene. Catal. Commun. 8, 2007-2011.

Nur, H., Guan, L. C., Endud, S., Hamdan, H. 2004. Quantitative measurement of a mixture of mesophases cubic MCM-48 and hexagonal MCM-41 by ${ }^{13}$ C CP/MAS NMR. Mater. Lett. 58, 12-13, 1971-1974.

Contescu, C. I. (2009). Dekker encyclopedia of nanoscience and nanotechnology. ( $2^{\text {nd }}$ Edition). Boca Raton, FL : CRC Press.

Riley, D. 1992. Industrial relations in Australian education in Contemporary Australasian industrial relations. Proceedings of the sixth AIRAANZ conference, ed. D Blackmur. 29 January-2 February 1992. Sydney, 124140.

Aghion, P., Durlauf, S. (eds). 2005. Handbook of economic growth, Amsterdam: Elsevier. Available from: Elsevier books. [4 November 2004].

Eringen, A. C. 1966. Theory of Micropolar Fluids. J. Math. Mech. 1-18.

Ariman, T., Turk, M. A. and Sylvester, N. D. 1973. Microcontinuum fluid mechanics-A Review. Inter. J. Eng. Sci. 905-930.

Eringen, A. C. (2001). Microcontinuum Field Theories II: Fluent Media. New York: Springer.

Lukaszewicz, G. (1999). Micropolar Fluids: Theory and Applications. Basel: Springer.

R. Nazar et al., 2002. Free convection boundary layer on an isothermal sphere in a micropolar fluid. Inter. Commun. Heat Mass Transf. 377-386.

Cheng, C. Y. 2008. Natural convection heat and mass transfer from a sphere in micropolar fluids with constant wall temperature and concentration. Inter. Commun.Heat Mass Transf. 750-755.

Sherief, H., Faltas, M. S. and Ashmawy, E. A. 2011. Exact solution for the unsteady flow of a semi-infinite micropolar fluid. Acta Mech. Sin. 354359.

El-Hakiem, M. A. 2014. Heat transfer from moving surfaces in a micropolar fluid with internal heat generation. J. Eng. Appl. Sci. 30-36.

Hassanien, I. A., Bakier, A. J. and Gorla, R. S. R. 1997. Natural convection boundary layer flow of a micropolar fluid. ZAMM, 751-755.

Ishak, A., Nazar, R. and Pop, I. 2008. Heat transfer over a stretching surface with variable heat flux in micropolar fluids. Phys. Lett. A, 559-561.

Lok, Y. Y., Amin, N., Campean, D. and Pop, I. 2005. Steady mixed convection flow of a micropolar fluid near the stagnation point on a vertical surface. Inter. J. Numer. Methods Heat Fluid Flow, 654-670.

Gorla, R. S. R. 1983. Micropolar boundary layer flow at stagnation on a moving wall. Inter. J. Eng. Sci. 25-33.

Damseh, R. A., Al-Azab, T. A., Shannak, B. A. and Husein, M. A. 2007. Unsteady natural convection heat transfer of micropolar fluid over a vertical surface with constant heat flux. Turk. J. Eng. Envir. Sci. 225-233.

Khalid, A., Khan, I., Khan, A. and Shafie, S. 2015. Conjugate transfer of heat and mass in unsteady flow of a micropolar fluid with wall couple stress. AIP Ad. 127125.

Abo-Dahab, S. M. and Mohamed, R. A. 2013. Unsteady flow of rotating and chemically reacting MHD micropolar fluid in slip-flow regime with heat generation. Inter. J. Thermophys. 2183-2208.

Chaudhary, R. C and Jain, A. 2007. Combined heat and mass transfer effects on MHD free convection flow past an oscillating plate embedded in porous medium. Rom. J. Phys. 505-524. 